\title{
Interactive comment on "Examining the link between vegetation leaf area and land-atmosphere exchange of water, energy, and carbon fluxes using FLUXNET data" by Anne J. Hoek van Dijke et al.
}

Anonymous Referee \#1

Received and published: 2 April 2020

The authors address the vegetation influence on interannual variability of surface energy and carbon fluxes. This topic is important for understanding ongoing land surface and climate changes affecting the water cycle, and related difficulties in numerical modeling. The study includes many sites and ecosystems globally, thus helping to fill some gaps in the literature. However, the manuscript could be revised so as to clarify the scope and generality of the results, and to provide additional analyses needed to support some of the conclusions. 
the paper is worded more broadly as a critique of how strongly water/energy/carbon fluxes are constrained by vegetation, and specifically stomatal control. It is unclear whether the weak constraint inferred at some sites or ecosystems is due to the LAI proxy missing some aspects of the vegetation influence, or if that influence is in fact negligible for some ecosystems (e.g., deciduous broadleaf forest). There is a practical issue in that LAI is often used where in-situ flux measurements of canopy-scale photosynthesis (of GPP or NEE, or some more direct measure of photosynthesis) are not available, and it is used in models to scale from the leaf to canopy - but land models account for many other aspects of vegetation that affect evapotranspiration beyond LAI. Thus some care is warranted to avoid setting up LAI in a 'straw man' argument. The question and problem statement could be clarified to be more about whether LAl is a good proxy for describing vegetation influences on water/energy fluxes, and when and where it is suitable for that purpose.

The study's focus is on interannual variability, but this is not reflected in the title and abstract. The choice of this timescale could also be better motivated in the introduction. We know that the seasonal variation in LAI is important for water/energy fluxes in most ecosystems and climates. The relationship between LAI and water/energy fluxes on interannual timescales is perhaps more subtle given relatively smaller interannual variations in LAI and (potentially) large variations between sites related to water-use efficiency or how efficiently plants use their leaves.

Major comment 2: The present study combines interannual variability and site-to-site variability which makes it difficult to interpret the results even when aggregated by ecosystem type. The lack of correlation between LAI and water/energy fluxes at interannual timescales could be due to such site variations. This would ideally be addressed with additional analyses to separate the two factors (site dependence and LAI), or at least could be acknowledged with a strongly worded caveat in the abstract and discussion/conclusions.

Printer-friendly version

\section{Discussion paper}

Detailed comments: Line 16: what does 'large-scale' mean in this context? Line 21:

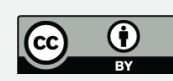


qualify that this is on annual average or interannual timescales Line 23: 'insight into'

Line 25: As noted above, the conclusion of the study needs as currently stated is more broadly worded than what the results and methods allow. Of course LAI is a necessary variable for modeling in order to scale photosynthesis and transpiration from leaf to canopy, so stating that it is not 'useful' is confusing. It may not be as helpful to consider LAI to be a 'parameter' either (line 64), in the sense of an adjustable factor or tuning knob. It is more like a variable that is either predicted or prescribed in order to model canopy-scale processes such as light interception. More specifically, what the authors seem to be saying is that LAI plays less of a role in explaining interannual variability of annually-averaged fluxes than other variables such as net radiation.

Line 30: Is the phrase "on the other hand" necessary or appropriate? Maybe "additionally" is more appropriate, since there is not a strong contrast between this sentence and what came before?

Line 53: Was the cited reference a modelling study, or an analysis of model output? There are other references in which LAI was experimentally changed in models to show what impact it has on climate predictions, which could also be cited here; for example Boussetta et al. 2013, but there are probably others.

Boussetta, Souhail, et al. "Impact of a satellite-derived leaf area index monthly climatology in a global numerical weather prediction model." International journal of remote sensing 34.9-10 (2013): 3520-3542.

Line 56: "indicative of"

Line 68: The discussion of saturation of NDVI is appreciated and relevant to the interpretation of forest results. There is also potentially a slight nonlinear saturation of the effect of LAI on EF and LH that may explain the weaker correlation between the two on interannual timescales.

Line 76: Again, I'm not sure what 'large-scale' means or what idea about scale the

Printer-friendly version

Discussion paper
Interactive comment 
authors are trying to convey. What would be considered small scale? Do you mean canopy scale, as opposed to leaf scale? Flux measurements are not what I consider to be 'large-scale' from a meteorological point of view. Those measurements typically need to be scaled up to be interpreted at the scale of a meteorological model grid cell $(100 \mathrm{~km})$.

Line 108: "In some land cover types, the surface fluxes and LAI showed seasonal variation." This statement understates the importance of the seasonal cycle. More realistically, most land cover types exhibit some kind of seasonal variation. Some sites may have muted seasonal variations, but even tropical sites have a wet and dry season.

Lines 110-114: I appreciate this discussion of the nonlinearity and what it means to average over the seasonal cycle. However it is still unclear how this coarse-scale temporal averaging affects the results and interpretation. For example, for deciduous broadleaf forests, the winter months are irrelevant for inferring the stomatal control on latent heat flux, so why include those months in the analysis if the goal is to quantify the vegetation influence on fluxes? Are the conclusions (that these sites show little vegetation or stomatal control on annually-averaged heat fluxes, based on correlations) dependent on the fact that for more than half of the year there is no active vegetation present?

Figure 3 - I'm assuming that there is a mistake and 'arid grassland' should have red markers, and 'humid grassland' should have blue. This figure could be described more clearly and with more information. What is meant by a 'moving window of aridity index'. What exactly do the markers represent? The caption mentions ' 30 site years..., and the paragraph (Line 165) mentions 'with a minimum of 15 site years for the lowest and highest aridity boundary), and figure itself shows about 20 data points for the humid and 23 for the arid, which is neither 15 nor 30 . My best guess is that all the site years were pooled within ecosystem types (mixing different sites into the same pool), and then ranked by aridity index. Then, the correlation between EF and LAI was calculated for the top and bottom 30 most humid and arid site years. But then why are there only

Printer-friendly version

Discussion paper

\section{(1)}


Another question is whether the top and bottom years ranked by aridity are dominated by a small subset of sites (i.e., sites with intermediate aridity are not shown in Fig. 3 ), and what impact the site-to-site variation has on the results. For example, some ecosystems may be more productive or have higher water-use efficiency than others for various reasons (soil type and nutrients, age of stand, amount of photosynthetically active radiation, etc) even within a given ecosystem type (grassland, forest, etc). I suspect that for each site, there is indeed a relationship between $L A I$ and $E F$, but the slope of that relationship is different for different sites even within the same vegetation type category. Some sites/species use their leaves more efficiently than others. If that were the case, then pooling all of the sites together could result in the weak relationships shown here. The 'all-year averages' shown in Fig. 6 indicate that most of the variation explored here is indeed due to variation across sites and not necessarily due to the variation in $\mathrm{LAl}$ alone.

Line 171: It would help to know whether this result holds when calculating the correlation separately for each site. Either way, the discussion of these results should mention this issue.

Figure 7: Consider better notation such as $\mathrm{r}(\mathrm{Flux}, \mathrm{P})$ to denote the correlation between the two, and likewise for $r(F l u x, R n)$, and then in the caption specify 'The correlation coefficient $(r)$ between surface fluxes and ...)'.

Line 230: There is some good discussion here on the role of canopy interception/evaporation, which one would think would contribute to a stronger relationship between LAI and LH or EF in forests, but as the authors noted this is not the case for temperate and boreal forest in this study. Again, the discussion is good, but it remains unclear why this study finds such a weak relationship and whether this is related to site variability and the chosen interannual timescale. It is also worth noting that the LAI derived from NDVI is "green" leaf area index, which is not necessarily the leaf

Printer-friendly version

Discussion paper 
area that is intercepting rainfall. There may be 'brown' leaves that participate in rainfall interception but result in a smaller 'green' LAI derived from NDVI.

Interactive comment on Biogeosciences Discuss., https://doi.org/10.5194/bg-2020-50, 2020. 